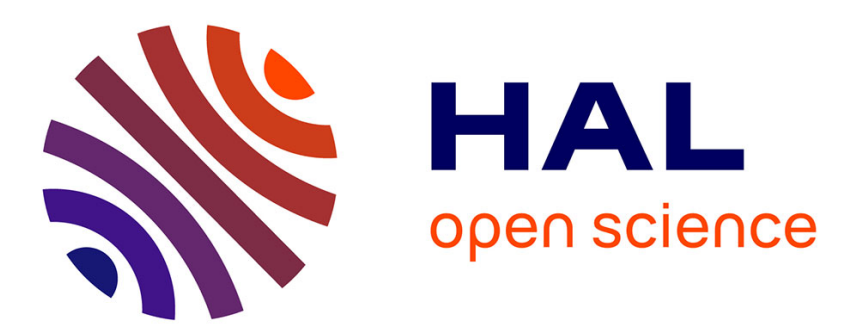

\title{
Rôle des paramètres physiques et géométriques sur la stabilité des composites supraconducteurs
}

B. Turck

\section{To cite this version:}

B. Turck. Rôle des paramètres physiques et géométriques sur la stabilité des composites supraconducteurs. Revue de Physique Appliquée, 1974, 9 (2), pp.503-514. 10.1051/rphysap:0197400902050300 . jpa-00243810

\section{HAL Id: jpa-00243810 https://hal.science/jpa-00243810}

Submitted on 1 Jan 1974

HAL is a multi-disciplinary open access archive for the deposit and dissemination of scientific research documents, whether they are published or not. The documents may come from teaching and research institutions in France or abroad, or from public or private research centers.
L'archive ouverte pluridisciplinaire HAL, est destinée au dépôt et à la diffusion de documents scientifiques de niveau recherche, publiés ou non, émanant des établissements d'enseignement et de recherche français ou étrangers, des laboratoires publics ou privés. 


\title{
Classification \\ Physics Abstracts \\ 8.490 \\ RÔLE DES PARAMẼTRES PHYSIQUES ET GÉOMÉTRIQUES SUR LA STABILITÉ DES COMPOSITES SUPRACONDUCTEURS
}

\author{
B. TURCK
}

\author{
Département du Synchrotron Saturne, \\ Service d'Entretien et de Développement des Appareils de Physique, \\ CEN, Saclay, BP 2, 91190 Gif-sur-Yvette, France
}

(Reçu le 27 août 1973, révisé le 26 octobre 1973)

\begin{abstract}
Résumé. - Les utilisateurs d'aimants supraconducteurs ont recours à différents types de stabilisation. Si, historiquement, les principes de la stabilisation cryostatique sont apparus les premiers, notre étude montre que ce système est loin d'être dépassé dans l'état actuel de réalisation des bobinages. On analyse, ici, le rôle des différents milieux environnant le supraconducteur en rappelant le rôle important de la perturbation initiatrice d'instabilité et en précísant les conditions de stabilité dans le cas d'une caractéristique réelle courant-tension.
\end{abstract}

\begin{abstract}
Engineers designing superconductive devices use several kinds of stabilization. If, historically, the cryostatic stabilization principle was used for the earliest magnets built, our study shows this criterion to be far from obsolescence. The influence of different surroundings of the superconductor is analyzed, pointing out the importance of the perturbation initiating the instability in the actual case of the current-voltage curve of the sample.
\end{abstract}

1. Introduction. - $\mathrm{Si}$ les critères de stabilité cryostatique, dynamique et adiabatique sont apparus historiquement à des dates différentes, il ne faut pas en déduire que le plus ancien serait forcément le plus démodé. Au contraire, il apparaît souvent que des conducteurs adiabatiquement ou dynamiquement stables, placés dans les conditions de fonctionnement des bobinages présentent une dégradation non négligeable [1]. On émet souvent l'hypothèse de mouvements possibles du conducteur soumis aux forces électromagnétiques, le frottement pouvant entraîner une élévation de température suffisante pour engendrer un «blocage » prématuré. Après avoir fait quelques remarques sur le rôle des "perturbations initiales", nous passons en revue les différents types d'environnement possibles du supraconducteur, en précisant dans chaque cas le critère à employer suivant les hypothèses limitatrices faites.

Une synthèse est ensuite réalisée avant de proposer pour un composite soumis à des perturbations extérieures, la notion de « stabilité conditionnelle ». Dans ce cas on utilise une caractéristique réelle couranttension non linéarisée.

2. Remarques générales. - 2.1 LES PERTURBATIONS ORIGINELLES. - On néglige généralement cette question, pour la bonne raison que l'on ne les connaît pas toujours. Seules les perturbations « endogènes » du type saut de flux peuvent être appréciées en énergie. Leur durée étant courte, on considère alors le système comme globalement adiabatique. Cependant, on sait qu'un conducteur " adiabatiquement " stable n'est pas protégé vis-à-vis des perturbations «extérieures ». En particulier, une élévation de température due à l'apport de chaleur produit par un mouvement et un frottement peut entraîner une dégradation importante. Dans ce cas, seule la stabilisation cryostatique totale est capable d'encaisser des perturbations importantes de température. Cependant, la situation peut être plus favorable pour plusieurs raisons.

a) Une partie de l'énergie de la perturbation peut être absorbée directement par enthalpie du matériau et de l'hélium si la durée est courte.

b) L'apparition de la tension pour un courant supérieur au «courant critique » se fait de manière très progressive.

c) Le transfert thermique du matériau vers l'hélium est meilleur en impulsion.

d) Les critères sont souvent déterminés en considérant que le phénomène affecte un conducteur infiniment long (étudié radialement) bien qu'en pratique il n'y a qu'une petite zone affectée. On peut, également, aborder la stabilité à partir de la recherche d'une propagation de perturbation à vitesse nulle. 
Par contre, dans le cas réel d'un bobinage l'environnement est très complexe (supraconducteur, cuivre, isolant, hélium, gaz, couches voisines, etc...) et l'obtention d'un critère global s'avère illusoire.

2.2 Quelques « CONSTANTES » PhysiQues. - Nous donnons pour quelques matériaux utilisés dans les bobinages supraconducteurs des constantes physiques telles qu'elles sont fournies dans la littérature. Des divergences importantes existent quelquefois, divergences pouvant être liées à l'état de pureté des échantillons (Tableau I).

$A$ masse atomique en $\mathrm{g}$;

$\delta$ masse spécifique en $\mathrm{g} / \mathrm{cm}^{3}$;

$T_{\mathrm{c}_{0}}$ température critique à $0 \mathrm{~K}$;

$H_{\mathrm{c} 1_{0}}, H_{\mathrm{c} 1_{4,2}} 1^{\mathrm{er}}$ champ critique à $0 \mathrm{~K}$ et $4,2 \mathrm{~K}$;

$H_{\mathrm{c} 2}, H_{\mathrm{c} 2{ }_{4,2}} 2^{\mathrm{e}}$ champ critique à $0 \mathrm{~K}$ et à $4,2 \mathrm{~K}$;

$\rho_{\mathrm{n}_{4,2}}$ résistivité à l'état normal à $4,2 \mathrm{~K}$;

$\rho_{\mathrm{n}_{300}}$ résistivité à l'état normal à $300 \mathrm{~K}$;

$K$ conductibilité thermique $\mathrm{W} / \mathrm{cm} \mathrm{K}$;

$C$ chaleur spécifique massique $\mathrm{J} / \mathrm{g} \mathrm{K}$;

$\lambda$ coefficient de retreint entre $300 \mathrm{~K}$ et $4,2 \mathrm{~K}$;

$Y$ limite élastique $\mathrm{kg} / \mathrm{mm}^{2}$.

Rappelons que la diffusivité thermique $D_{\theta}$ est égale à $K / \delta C$ et que la diffusivité magnétique $D m$ est égale à $\rho_{\mathrm{n}} / \mu_{0}$.

A ce tableau, il faut ajouter la chaleur latente de vaporisation de l'hélium à $4,2 \mathrm{~K}$ soit $L=24,5 \mathrm{~J} / \mathrm{g}$.

2.3 Vitesses De propagation de La Chaleur a TRAVERS DES MATÉRIAUX. - Dans le cas d'un bobinage, le comportement en température dépend en grande partie de la diffusivité thermique des matériaux.

Si la diffusivité est grande, c'est la conductibilité qui va régir le problème et le phénomène doit être étudié en fonction du temps ; on parlera puissance. $\mathrm{Si}$ par contre elle est petite, c'est essentiellement la chaleur spécifique qui intervient et pour des perturba- tions de courte durée, on peut parler d'énergie à dissiper plutôt que de puissance [2].

L'évacuation de chaleur peut se faire dans plusieurs directions. A la distance $x$ de la source, elle se fait :

- Longitudinalement : par le cuivre avec une vitesse

$$
V \simeq \frac{0,5}{x} \mathrm{~m} / \mathrm{s} .
$$

- Radialement :

- par l'isolant avec

$$
V \simeq \frac{2 \times 10^{-5}}{x} \mathrm{~m} / \mathrm{s}
$$

— par l'hélium gaz avec

$$
V \simeq \frac{2 \times 10^{-7}}{x} \mathrm{~m} / \mathrm{s}
$$

- par l'hélium liquide avec

$$
V \simeq \frac{5 \times 10^{-8}}{x} \mathrm{~m} / \mathrm{s} .
$$

2.4 COURBes DE TRANSFERT CUIVRE-HÉLIUM. Un rôle fondamental est joué par le transfert de la chaleur du cuivre vers l'hélium. Si la chaleur latente de vaporisation est élevée, la puissance évacuée est vite limitée par la surface de contact et la conductibilité thermique du liquide et des bulles de gaz formées. Il est utile de considérer les possibilités de l'échange sous la forme d'une courbe de transfert reliant la puissance concernée par unité de surface mouillée à l'écart de température entre le bain et le matériau. Suivant cet écart, on distingue trois régimes caractéristiques, de convection d'ébullition nucléée et d'ébullition en film [3].

\begin{tabular}{|c|c|c|c|c|c|c|c|c|c|c|c|c|c|c|c|c|c|}
\hline Corps & A & $\delta$ & $T c_{0}$ & $\begin{array}{l}\mathrm{Hc}_{1} \\
0^{\circ} \mathrm{K}\end{array}$ & $\mid \begin{array}{c}\mathrm{Hc} \\
4,2^{\circ} \mathrm{K}\end{array}$ & $\begin{array}{l}\mathrm{Hc} c_{2} \\
0^{\circ} \mathrm{K}\end{array}$ & $\begin{array}{l}\mathrm{Hc}_{2} \\
4,2^{\circ} \mathrm{K}\end{array}$ & $\begin{array}{c}\rho^{\circ} \\
4,2^{\circ} \mathrm{K}\end{array}$ & $\begin{array}{c}P_{n} \\
300^{\circ} \mathrm{K}\end{array}$ & $\begin{array}{c}\mathrm{K} \\
4,2^{\circ} \mathrm{K}\end{array}$ & $\begin{array}{c}\mathrm{C} \\
4,2 \circ \mathrm{K}\end{array}$ & $\begin{array}{c}\mathrm{C} \\
10^{\circ} \mathrm{K}\end{array}$ & $\begin{array}{c}\mathrm{C} \\
20^{\circ} \mathrm{K}\end{array}$ & $\lambda$ & $\begin{array}{c}\mathrm{Y} \\
4,2^{\circ} \mathrm{K}\end{array}$ & $\begin{array}{c}\mathrm{Y} \\
300^{\circ} \mathrm{K}\end{array}$ & Corps \\
\hline $\begin{array}{l}\text { Cuivre } \\
\text { Aluminium } \\
\text { Cupro Nickel } \\
\text { Acıer Inox } \\
\text { Indium } \\
\text { Plomb } \\
\text { Etain } \\
\text { Soudure } \mathrm{Pb} / \mathrm{Sn}\end{array}$ & $\begin{array}{r}115 \\
207 \\
118\end{array}$ & $\begin{array}{c}8,92 \\
2,7 \\
8,6 \\
7,9 \\
7,3 \\
11,3 \\
6 \\
9,5\end{array}$ & $\begin{array}{l}3,4 \\
7,2 \\
3,7\end{array}$ & $\begin{array}{l}285 \\
800 \\
300\end{array}$ & & & - & $\begin{array}{c}0,01 \\
0,01 / 0,1 \\
40 \\
0,1 / 10 \\
0,02 \\
0,004 \\
0,01\end{array}$ & $\begin{array}{c}1,7 \\
2,8 \\
50 \\
10 / 20 \\
8,4 \\
22 \\
11,5 \\
16\end{array}$ & \begin{tabular}{|c|}
3000 \\
$500 / 30000$ \\
$10 / 40$ \\
2,5 \\
8400 \\
20000 \\
25000 \\
150
\end{tabular} & $\begin{array}{l}0,1 \\
0,25 \\
0,4 \\
0,4 \\
0,1 \\
0,7 \\
0,3 \\
0,4\end{array}$ & $\begin{array}{l}0,8 \\
1,5 \\
1,2 \\
15 \\
11 \\
10 \\
11\end{array}$ & $\begin{array}{l}4,5 \\
60 \\
50 \\
40\end{array}$ & $\begin{array}{l}0,3 \\
0,7 \\
0,7 \\
0,7 \\
0,7\end{array}$ & $\begin{array}{l}7 / 50(40) \\
3 / 80(40)\end{array}$ & \begin{tabular}{|c|}
$7 / 30(20)$ \\
$6 / 30(12)$ \\
35 \\
$25 / 110$ \\
\\
2 \\
$2,5 / 7$ \\
7
\end{tabular} & $\begin{array}{l}\text { Cuivre } \\
\text { Aluminium } \\
\text { Cupro Nickel } \\
\text { Acier Inox } \\
\text { Indium } \\
\text { Plomb } \\
\text { Etain } \\
\text { Soudure } \mathrm{Pb} / \mathrm{Sn}\end{array}$ \\
\hline $\begin{array}{l}\mathrm{NbT} \\
\mathrm{Nb}_{3} \mathrm{Sn} \\
\mathrm{V}_{3} \mathrm{go}\end{array}$ & & $\begin{array}{l}5,6 \\
5,4\end{array}$ & $\left|\begin{array}{r}9,5 \\
18,2 \\
16,8\end{array}\right|$ & $\begin{array}{l}140 \\
200 \\
400\end{array}$ & & $\begin{array}{l}125000 \\
260000 \\
250000\end{array}$ & $\begin{array}{l}110000 \\
220000 \\
200000\end{array}$ & $\begin{array}{l}24 \\
20 \\
40\end{array}$ & & $\begin{array}{c}1,2 \\
4 \\
0,4\end{array}$ & $\begin{array}{c}0,2 / 0,7 \\
0,35\end{array}$ & & & 0,13 & & 70 & $\begin{array}{l}\mathrm{Nb} T_{1} \\
\mathrm{Nb}_{3} \mathrm{Sn} \\
\mathrm{V}_{3} \mathrm{ga}\end{array}$ \\
\hline $\begin{array}{l}\text { Verre } \\
\text { Epoxy } \\
\text { Téflon } \\
\text { Nylon } \\
\text { He liquide } \\
\text { He gaz }\end{array}$ & $\begin{array}{l}4 \\
4\end{array}$ & $\begin{array}{c}2,5 \\
2,1 \\
0,125 \\
0,008\end{array}$ & - &.- & & - & {$\left[\begin{array}{l}-\cdots \\
- \\
-\end{array}\right.$} & & $\begin{array}{c}10^{18} / 10^{21} \\
10^{22} / 10^{23} \\
10^{21} \\
4.10^{20}\end{array}$ & 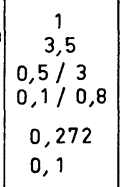 & $\begin{array}{c}0,2 \\
2 \\
2 \\
\\
4500 \\
6000\end{array}$ & $\begin{array}{l}4 \\
30 \\
18\end{array}$ & $\begin{array}{l}27 \\
80 \\
75 \\
\end{array}$ & $\begin{array}{l}0,1 \\
1,1 \\
2,1 \\
1,4\end{array}$ & $\begin{array}{l}7,5 \\
70 \\
15 \\
25\end{array}$ & $\begin{array}{c}6 \\
25 / 50 \\
1,5 \\
7\end{array}$ & $\begin{array}{l}\text { Verre } \\
\text { Epoxy } \\
\text { Téflon } \\
\text { Nylon } \\
\text { He liquide } \\
\text { He gaz }\end{array}$ \\
\hline Unités & $g$ & $\mathrm{gk \textrm {km } ^ { 3 }}$ & ${ }^{\circ} \mathrm{K}$ & Gauss & Gauss & Gouss & Gauss & $\begin{array}{c}10^{-6} \\
\text { ohm cm }\end{array}$ & $\begin{array}{c}10^{-6} \\
\text { ohm.cm }\end{array}$ & $\begin{array}{c}10^{-3} \\
\mathrm{~W} / \mathrm{cm}{ }^{\circ} \mathrm{K}\end{array}$ & $\begin{array}{l}10^{-3} \\
J / 9^{\circ} \mathrm{K}\end{array}$ & $\begin{array}{c}10^{-3} \\
\mathrm{~J} / \mathrm{g}^{\circ} \mathrm{K}\end{array}$ & $\begin{array}{l}10^{-3} \\
\mathrm{~J} / \mathrm{g}^{\circ} \mathrm{K}\end{array}$ & $\%$ & $\mathrm{~kg} / \mathrm{mm}^{2}$ & $\mathrm{~kg} / \mathrm{mm}^{2}$ & Unités \\
\hline
\end{tabular}

Nous présentons figure 1 , trois courbes de transfert cuivre-hélium.

TABleau I 


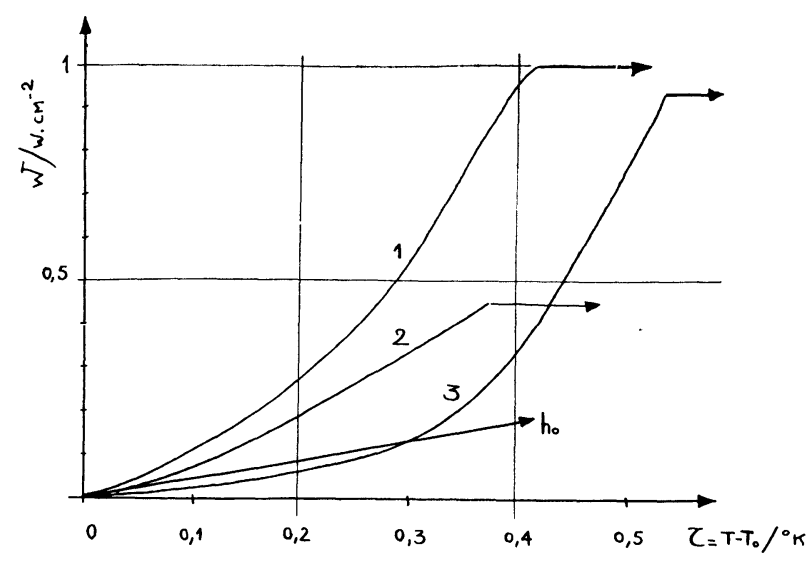

FIG. 1. - Courbes de transfert cuivre-hélium : Courbe 1, fil rond horizontal; Courbe 2, ruban épais horizontal $(10 \times 1,75 \mathrm{~mm})$; Courbe 3 , fil rond vertical d'après $\mathrm{K}$. R. Efferson [5] ( $h_{0}$ est la pente à l'origine choisie).

Les deux premières mesurées dans notre laboratoire [4] correspondent à un fil rond, et à un ruban placés horizontalement. La température a été déduite de la décroissance du courant critique avec la température.

La troisième est la courbe correspondant à un fil vertical, fournie par K. R. Efferson [5].

2.5 Apparition de LA tension. Notion de COURANT CRITIQUE. - Lorsque l'on fait croître le courant dans un échantillon composite supraconducteur cuivre, une tension apparaît progressivement. Si la température du matériau supposé isotherme reste celle du bain d'hélium, on peut distinguer deux régions :

- La première où la loi $v(I)$ semble proche d'une exponentielle (transferts de courant multiples de brin à brin à travers le cuivre); où les résistivités $(E / J)$ apparentes sont de l'ordre de $10^{-16} \Omega$.m et les résistivités différentielles $(\mathrm{d} E / \mathrm{d} J)$ de l'ordre de $10^{-12} \Omega$.m.

- La deuxième où le champ électrique est tel que le supraconducteur est entré nettement en régime de flot de flux $\left(\rho_{\mathrm{f}} \simeq 2 \times 10^{-7} \Omega\right.$.m). Le courant se partage alors entre cuivre et supraconducteur. La résistivité du cuivre étant de $10^{-10} \Omega . \mathrm{m}$, pratiquement tout le courant supplémentaire passe dans celui-ci (Fig. 2).

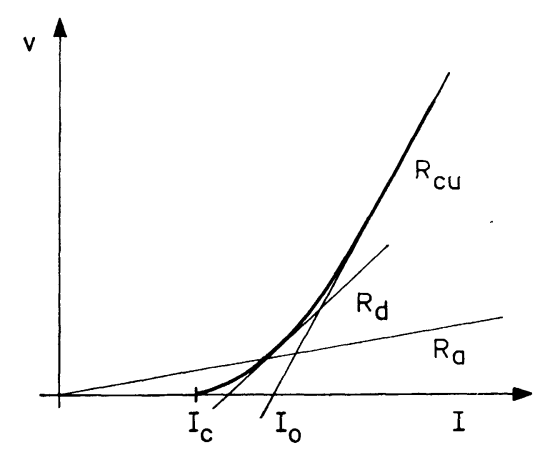

FIG. 2. - Caractéristiques courant-tension d'un échantillon composite $R_{\mathrm{a}}, R_{\mathrm{d}}$ et $R_{\mathrm{cu}}=$ résistances apparente, différentielle et résistance du cuivre.
Dans la première région on peut définir un courant dit « critique » correspondant à un champ électrique donné (par exemple $0,25 \mu \mathrm{V} / \mathrm{cm}$ ) ou à une résistivité apparente donnée (par exemple $4 \times 10^{-14} \Omega$.m) [6].

Dans cette zone, la résistivité différentielle est bien inférieure à celle du cuivre et nous verrons plus loin (Chap. 9) comment tenir compte de cette question. Par contre pour la suite des calculs, nous considérerons le cas simplifié, où la tension reste nulle tant que le courant reste inférieur à $I_{0}$. Ensuite, elle croît avec une pente correspondant à la résistance du cuivre stabilisant, en parallèle sur le supraconducteur.

Ainsi si $I$ est le courant total dans le composite, à la température du bain $T_{0}$, de $4,2 \mathrm{~K}$ on prend :

$$
\text { pour } I<I_{0} \quad V=0
$$

et

$$
\text { pour } I>I_{0} \quad V=R_{\mathrm{cu}}\left(I-I_{0}\right) \text {. }
$$

Lorsque la température n'est plus celle du bain d'hélium, il faut tenir compte de la décroissance du courant "critique " avec la montée en température. Celle-ci est approximativement linéaire, et si l'on appelle ici $T_{\mathrm{c}}$ la température correspondant à l'extrapolation vers zéro de la partie linéaire de la loi $J_{\mathrm{c}}(T)$ on peut écrire :

$$
J_{\mathrm{s}}=J_{0}\left(1-\frac{\mathfrak{C}}{\mathfrak{G}_{\mathrm{c}}}\right)
$$

$J_{\mathrm{s}}$ est la densité de courant dans le supraconducteur à la température $T$, lorsque $J_{0}$ est la densité à la température $T_{0}$, correspondant au courant $I_{0}$. De plus on appelle $\mathcal{C}$ l'écart de température $T-T_{0}$ au-dessus de la température du bain d'hélium.

Nous utiliserons toujours cette quantité $\mathcal{C}$ plutôt que la température absolue $T$.

3. Définition de la stabilisation cryostatique. Si les premières études ont porté sur ce type de stabilisation [7], [8], [9], des calculs plus ou moins élaborés se poursuivent à l'heure actuelle [4], [10], [11], en particulier pour la stabilisation de gros bobinages à énergie stockée élevée. Si les principes mis en cause sont loin d'être dépassés, c'est que ce type de stabilisation est capable d' "encaisser " les fortes perturbations.

3.1 Comme son nom l'indique, on n'étudie pas l'évolution de la température avec le temps. On détermine, tout régime transitoire terminé, le régime d'équilibre permanent établi entre courant, température, puissance, etc...

3.2 On étudie le comportement du composite au voisinage du courant critique, en observant le partage de courant entre le cuivre et le supraconducteur, soit lorsque la température est supérieure à $T_{0}$, soit lorsque le courant est supérieur à $I_{0}$. Suivant l'échange de puissance avec le milieu extérieur, il s'établit un régime 
où selon la valeur trouvée pour la température on peut estimer que le composite est stable ou instable.

3.3 Cette protection cryostatique est, en général, sévère mais sûre car elle ne suppose rien de l'origine de la perturbation qu'elle soit exogène ou endogène (mouvement ou saut de flux).

4. Condition de stabilité fondamentale avec le transfert à l'hélium. - 4.1. EQUATION DE BASE DE L'ÉCHANGE. - Les calculs sont effectués par unité de longueur de conducteur. En supposant le supraconducteur isotherme, en considérant le composite non isolé baignant parfaitement dans l'hélium, au courant total $I$, à la température $T=T_{0}+\mathcal{G}$, une partie $I-I_{\text {s }}$ de courant circule dans le cuivre, la puissance totale à dissiper est :

$$
W=R_{\mathrm{cu}}\left(I-I_{\mathrm{s}}\right) I
$$

$I_{\mathrm{s}}$ est le courant dans le supraconducteur

$$
I_{\mathrm{s}}=I_{0}\left(1-\frac{\mathfrak{C}}{\mathscr{C}_{\mathrm{c}}}\right) \text {. }
$$

Cette puissance définit une droite de charge en fonction du courant total $I$ et de la température

$$
W=R_{\mathrm{cu}} I\left(I-I_{0}+I_{0} \frac{\mathfrak{C}}{\mathfrak{G}_{\mathrm{c}}}\right) \text {. }
$$

Nous discutons la stabilité dans le cas où l'on fait fonctionner le composite à un courant $I$, le plus voisin possible de $I_{0}$. Pour les autres cas où $I<I_{0}$, la stabilité est conditionnellement liée à l'amplitude de la perturbation initiale en température et la recherche des conditions se rapproche de celle, faite au chapitre 9.

Pour $I=I_{0}$, on peut donc écrire :

$$
W=R_{\mathrm{cu}} I_{0}^{2} \frac{\mathfrak{C}}{\mathscr{C}_{\mathrm{c}}}
$$

Cette puissance n'apparaît pour $\mathscr{C}$ petit, pratiquement que dans le supraconducteur.

Ainsi une augmentation de température $\mathscr{C}$ éjecte une partie $I-I_{\text {s }}$ de courant du supraconducteur, qui s'installe dans le cuivre. Un régime d'équilibre s'établit en fonction des possibilités d'évacuation de la chaleur vers l'hélium, c'est-à-dire par l'intermédiaire de la courbe de transfert cuivre-hélium.

Le point de fonctionnement est donné par l'intersection de la droite de charge avec cette courbe de transfert. La condition de stabilité exige donc que la pente de cette droite soit inférieure à la pente à l'origine $h_{0}$ de la courbe de transfert [4] (Fig. 3).

Dans le cas contraire, il peut y avoir :

- instabilité totale si la droite de charge est toujours au-dessus de la courbe de transfert,

- instabilité partielle si la droite de charge coupe la courbe de transfert. Dans ce cas, lorsque le courant atteint $I_{0}$, il y a saut brutal du point de fonctionnement

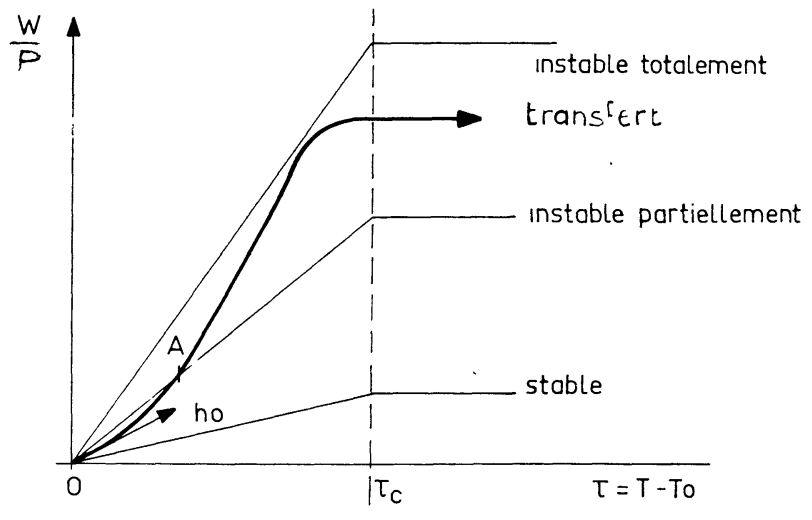

FIG. 3. - Transfert à l'hélium et stabilité pour $I=I_{0}$.

de $\mathrm{O}$ à $\mathrm{A}$, mais avec apparition de tension et dissipation de puissance non catastrophiques. D'où, la condition classique :

$$
\frac{\mathrm{d} W}{\mathrm{~d} \mathscr{C}}<h_{0} P
$$

soit

$$
\frac{R_{\mathrm{cu}} I_{0}^{2}}{\mathfrak{G}_{\mathrm{c}}}<h_{0} P
$$

$P$ étant le périmètre mouillé.

De cette relation on peut faire apparaître un critère de stabilité $\beta_{\mathrm{t}}$ dû au transfert, que l'on peut écrire $\beta_{\mathrm{t}}<1$. L'expression (5) s'écrit en effet :

$$
\frac{R_{\mathrm{cu}} I_{0}^{2}}{\mathfrak{G}_{\mathrm{c}}} \cdot \frac{1}{h_{0} P}<1 \text {. }
$$

On s'aperçoit que $\beta_{\mathrm{t}}$ n'est autre que le rapport entre les incréments de puissance générée dans le composite et de puissance évacuable à l'hélium liquide pour une élévation de température $\mathrm{d} \mathscr{C}$ infinitésimale.

En appelant $X$ le rapport des sections de cuivre et de supraconducteur, et si l'on suppose le composite fait de $N$ brins supraconducteurs de rayon $r_{\mathrm{s}}$, si $R$ est le rayon du cuivre extérieur, en posant $R_{\mathrm{s}}=r_{\mathrm{s}} \sqrt{N}$ (rayon d'un monobrin supraconducteur de même section) le coefficient de stabilité $\beta_{\mathrm{t}}$ s'écrit plus généralement :

$$
\beta_{\mathrm{t}}=\frac{\rho_{\mathrm{cu}} J_{0}^{2} R}{2 X(1+X) h_{0} \mathscr{C}_{\mathrm{c}}}
$$

ou encore :

$$
\beta_{\mathrm{t}}=\frac{\rho_{\mathrm{cu}} J_{0}^{2} R_{\mathrm{s}}}{2 X \sqrt{1+X} h_{0} \mathcal{G}_{\mathrm{c}}} .
$$

Certains auteurs [2], [12], [13] considèrent plutôt pour condition de stabilité que dans l'hypothèse d'un blocage total (tout le courant dans le cuivre), la puissance totale dissipée ne doit pas dépasser un certain seuil $W_{\mathrm{s}}$, par exemple la puissance de transition nucléée-film, ou la puissance de récupération film- 
TABleAU II

\begin{tabular}{|c|c|c|c|c|}
\hline Conducteur & $\begin{array}{c}\text { CFTH } \\
\text { BIM } \\
-\end{array}$ & $\begin{array}{c}\text { CFTH } \\
\text { B } 11 \\
-\end{array}$ & $\begin{array}{c}\text { IMI } \\
61-33 \\
-\end{array}$ & $\begin{array}{c}\text { IMI } \\
1000-44 \\
-\end{array}$ \\
\hline$N$ brins & 30 & 61 & 61 & 1000 \\
\hline$X=\frac{A_{\mathrm{cu}}}{A_{\mathrm{s}}}$ & 8 & 2 & 1,3 & 1 \\
\hline$\Phi_{\text {ext }}(\mathrm{mm})$ & $\begin{array}{c}\text { (ruban plat) } \\
P=23,5 \mathrm{~mm}\end{array}$ & 0,6 & 0,33 & 0,44 \\
\hline$\varphi_{\mathrm{s}}$ brin (mm) supra. & 0,3 & 0,043 & 0,028 & 0,010 \\
\hline$J_{0} / \mathrm{A} \cdot \mathrm{cm}^{-2}\left\{\begin{array}{l}1 \mathrm{~T} \\
6 \mathrm{~T}\end{array}\right.$ & $\begin{array}{r}5 \times 10^{5} \\
10^{5}\end{array}$ & $\begin{array}{r}6 \times 10^{5} \\
1,6 \times 10^{5}\end{array}$ & $\begin{array}{l}3,3 \times 10^{5} \\
1,2 \times 10^{5}\end{array}$ & $\begin{array}{r}3,5 \times 10^{5} \\
1,27 \times 10^{5}\end{array}$ \\
\hline
\end{tabular}

bulle. Dans ce dernier cas on dit que le conducteur est hyperstabilisé. Le critère s'écrit alors :

$$
\frac{\rho_{\mathrm{cu}} J_{0}^{2} R}{2 X(1+X) W_{\mathrm{s}}}<1 \text {. }
$$

Cette condition est, en général, plus sévère que la précédente, mais tout dépend essentiellement de la courbe de transfert. Ainsi dans le cas de la figure 4, le conducteur satisfait le critère ci-dessus (c'est-à-dire $\left.R_{\mathrm{cu}} I_{0}^{2}<W_{\mathrm{t}} / P\right)$, mais il y a instabilité partielle à l'origine. Ce cas est possible pour les champs très forts (6 à $7 \mathrm{~T})$.

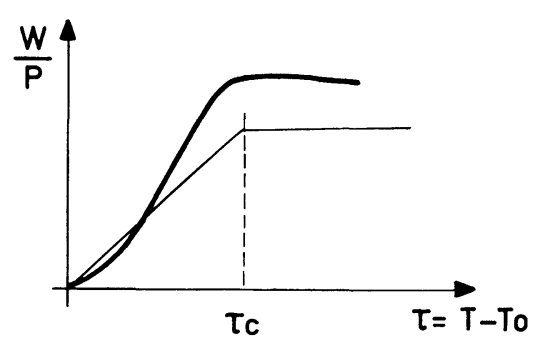

FIG. 4. - Cas possible d'instabilité.

On voit ainsi l'intérêt des conducteurs de dimensions extérieures petites de manière à augmenter l'échange par l'intermédiaire d'un rapport périmètre mouillé sur section, élevé.

4.2 ApPlication NUmÉRIQUe. - Nous avons choisi quatre types de conducteur auxquels nous ferons toujours référence.

Nous prendrons à $1 \mathrm{~T}$

$$
\rho_{\mathrm{cu}} \sim 2 \times 10^{-10} \Omega \text {.m } \text { et } \mathscr{C}_{\mathrm{c}} \sim 4 \mathrm{~K}
$$

à $6 \mathrm{~T}$

$$
\rho_{\mathrm{cu}} \sim 4,5 \times 10^{-10} \Omega . \mathrm{m} \text { et } \zeta_{\mathrm{c}} \sim 2 \mathrm{~K} .
$$

Le tableau II en résume les propriétés essentielles.
Le tableau III donne les valeurs de coefficient de stabilité $\beta_{\mathrm{t}}$ calculé à partir de ces valeurs, pour une pente $h_{0}$ à l'origine de la courbe de transfert, égale à $0,4 \mathrm{~W} / \mathrm{cm}^{2} \mathrm{~K}$ conformément aux mesures effec-

\begin{tabular}{|c|c|c|c|c|}
\hline Conducteur & BIM & B 11 & $61-33$ & $1000-44$ \\
\hline${ }_{B}\{1 \bar{T}$ & $\overline{3,5}$ & $\overline{11}$ & $\overline{3,7}$ & 8,5 \\
\hline$\beta_{\mathrm{t}}\{6 \mathrm{~T}$ & 0,6 & 3,3 & 2,2 & 5 \\
\hline
\end{tabular}
tuées [4].

\section{TABLEAU III}

Ainsi, seul le conducteur BIM est stable cryostatiquement à $6 \mathrm{~T}$, ce qui est d'ailleurs confirmé par l'expérience [14]. Nous verrons plus loin ce qui peut être dit des autres.

Le coefficient $\beta_{\mathrm{t}}$ indiqué donne aussi une mesure de l'instabilité du matériau. Plus $\beta_{\mathrm{t}}$ est grand, plus le matériau est instable. On peut aussi en déduire le rapport cuivre/supraconducteur nécessaire à la stabilité ou le courant admissible.

4.3 Abaque De stabilité CRyostatique liée aU TRANSFERT. - Il peut être intéressant de tracer un abaque de stabilité permettant de situer un conducteur donné en fonction de ses dimensions, dans une zone de stabilité ou d'instabilité. A cette fin, on réécrit la condition $\beta_{\mathrm{t}}<1$, sous la forme :

$$
\frac{\rho_{\mathrm{cu}} J_{0}^{2}}{h_{0} b_{\mathrm{c}}}<\frac{2 X \sqrt{1+X}}{R_{\mathrm{s}}}
$$

ou encore avec $\gamma=\frac{\Phi_{\text {ext }}}{\Phi_{\mathrm{s}}}$

$$
\Phi_{\mathrm{s}}<\frac{4 \gamma\left(\gamma^{2}-1\right) h_{0} \ell_{\mathrm{c}}}{\rho_{\mathrm{cu}} J_{0}^{2}} \text {. }
$$


En prenant : $h_{0}=0,4 \mathrm{~W} / \mathrm{cm}^{2} \mathrm{~K}$, ainsi que les densités moyennes

$$
J_{0_{1}} \simeq 4 \times 10^{5} \mathrm{~A} / \mathrm{cm}^{2} \text { à } 1 \mathrm{~T}
$$

et

$$
J_{0_{6}} \simeq 1,3 \times 10^{5} \mathrm{~A} / \mathrm{cm}^{2} \text { à } 6 \mathrm{~T},
$$

nous avons tracé (Fig. 5) l'abaque de stabilité et reporté les points correspondant aux quatre conducteurs choisis. Un conducteur est stable si son affixe est au-dessus de la courbe. Inversement, plus il en est éloigné par en dessous, plus il est instable cryostatiquement.

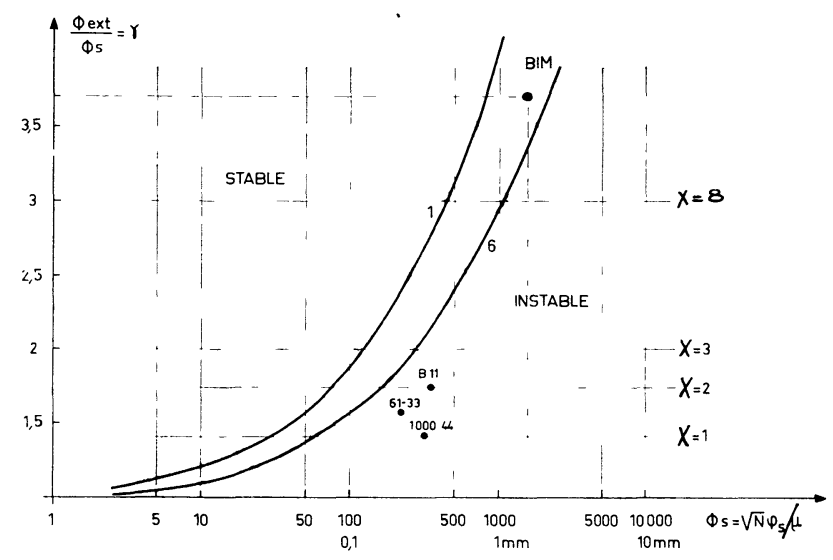

FIg. 5. - Abaque de stabilité cryostatique.

4.4 EXTENSION AU CAS D'UN ASSEMBLAGE DE CONDUCTEURS. - Dans ce cas, une perturbation en température provenant par exemple d'un frottement, peut n'affecter qu'un nombre limité $k$ de conducteurs parmi les $N$ de l'assemblage, pourvu que la propagation de la chaleur se fasse difficilement. A la différence du conducteur massif de même diamètre extérieur apparent, un transfert de courant peut s'effectuer d'un conducteur à l'autre. Un conducteur affecté peut en un sens bénéficier de l'ensemble du cuivre. C'est le cas des conducteurs assemblés recouverts d'une fine couche d'oxyde qui semblent présenter de gros avantages vis-à-vis de la stabilité. Dans le cas où seulement $k$ conducteurs parmi $N$ seraient affectés, on peut montrer [15] que la stabilité est assurée par la relation :

$$
\frac{\rho_{\mathrm{cu}} J_{0}^{2} R}{2 X(1+X) h_{0} \mathscr{G}_{\mathrm{c}}} \cdot \frac{k}{N}<1 .
$$

5. Influence de l'épaisseur des brins supraconducteurs. - 5.1 DÉTERMINATION DE LA TEMPÉRATURE DU SUPRACONDUCTEUR. - Nous supposons tout d'abord dans ce chapitre 5 , que le cuivre reste isotherme quelle que soit la puissance qui s'y dissipe, par contre il n'en est plus de même dans le supraconducteur. L'apparition d'une tension aux bornes du composite lors du partage du courant entre le supraconducteur et le cuivre, entraîne l'existence d'une puissance dissipée dans le supraconducteur même. Il apparaît alors dans chaque brin élémentaire un gradient thermique fonction de cette puissance.

Localement dans chaque brin la densité de courant $J_{\mathrm{s}}(r)$ et la température $\mathcal{C}(r)$ sont étroitement liées par la relation :

$$
J_{\mathrm{s}}(r)=J_{0}\left(1-\frac{\mathscr{C}}{\mathscr{G}_{\mathrm{c}}}\right)
$$

ainsi que

$$
\frac{\partial J_{\mathrm{s}}}{\partial r}=-\frac{J_{0}}{\mathfrak{G}_{\mathrm{c}}} \cdot \frac{\partial \mathscr{C}}{\partial r} .
$$

Si $v$ est le champ électrique longitudinal et $p_{\mathrm{s}}(r)$ la densité de puissance, l'évacuation de la chaleur vers l'extérieur se fait en fonction de la conductibilité thermique du supraconducteur et du gradient thermique local suivant la relation :

$$
-2 \pi r K_{\mathrm{s}} \frac{\partial \mathscr{C}}{\partial r}=\int_{0}^{r} 2 \pi r p_{\mathrm{s}}(r) \mathrm{d} r
$$

avec

$$
p_{\mathrm{s}}(r)=J_{\mathrm{s}}(r) \cdot v .
$$

De cette relation, on tire l'équation fondamentale

$$
\frac{\partial^{2} \mathscr{C}}{\partial r^{2}}+\frac{1}{r} \frac{\partial \mathscr{C}}{\partial r}+\frac{1}{K_{\mathrm{s}}} v J_{0}\left(1-\frac{\mathscr{C}}{\mathscr{C}_{\mathrm{c}}}\right)=0 .
$$

Si l'on pose $\alpha=v J_{0} / K_{\mathrm{s}} \mathscr{C}_{\mathrm{c}}$, on peut écrire les solutions locales de courant et de température en fonction de la température $\mathscr{C}_{2}$ au centre du brin

$$
\begin{aligned}
\mathfrak{C} & =\mathscr{C}_{\mathrm{c}}+\left(\mathscr{C}_{2}-\mathscr{C}_{\mathrm{c}}\right) \mathfrak{J}_{0}(r \sqrt{\alpha}) \\
J_{\mathrm{s}}(r) & =J_{0}\left(1-\frac{\mathfrak{C}_{2}}{\mathfrak{C}_{\mathrm{c}}}\right) \mathfrak{J}_{0}(r \sqrt{\alpha}) .
\end{aligned}
$$

Les fonctions $\mathfrak{J}_{0}$ et $\mathfrak{J}_{1}$ apparaissant plus loin sont les fonctions de Bessel modifiées de première espèce.

Il faut maintenant rapporter ces expressions aux quantités macroscopiques du composite en particulier $I_{\mathrm{s}}$ courant total circulant dans les $N$ brins supraconducteurs. $I_{\mathrm{s}}$ n'est autre que :

$$
I_{\mathrm{s}}=N \int_{0}^{r_{\mathrm{s}}} 2 \pi r J_{\mathrm{s}}(r) \mathrm{d} r
$$

soit

$$
I_{\mathrm{s}}=\frac{2 \pi}{v} \mathfrak{C}_{\mathrm{c}} N K_{\mathrm{s}}\left(1-\frac{\mathfrak{C}_{2}}{\mathscr{C}_{\mathrm{c}}}\right) r_{\mathrm{s}} \sqrt{\alpha} J_{1}\left(r_{\mathrm{s}} \sqrt{\alpha}\right) .
$$

Pourvu que la dimension $r_{\mathrm{s}}$ soit suffisamment petite (ce qui est le cas pour les composites étudiés), en développant au $2^{\mathrm{e}}$ ordre les fonctions de Bessel, on 
exprime la température $\mathfrak{C}_{1}$ au bord du supraconducteur (et dans le cuivre) [4], [10] figure 6

$$
\frac{\mathfrak{C}_{1}}{\mathfrak{G}_{\mathrm{c}}}=1-\frac{I_{\mathrm{s}}}{I_{0}}\left[1+\frac{v I_{0}}{v I_{0}+8 \pi K_{\mathrm{s}} N \mathscr{C}_{\mathrm{c}}}\right]
$$

avec $v=R_{\mathrm{cu}}\left(I-I_{\mathrm{s}}\right)$.

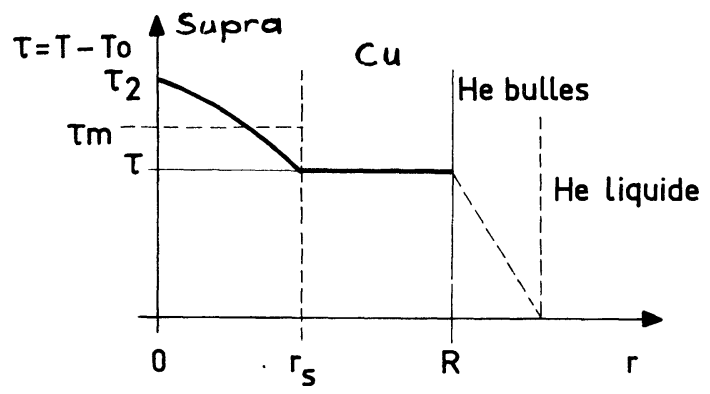

FIG. 6. - Répartition de température dans le composite.

5.2 RECHERCHE DU POINT DE FONCTIONNEMENT. Pour la compréhension du phénomène et la détermination du point de fonctionnement et des conditions de stabilité, il est bon de rappeler les expressions générales des puissances respectives dans le cuivre et le supraconducteur par unité de longueur de composite

$$
\begin{aligned}
& W_{\mathrm{cu}}=v I_{\mathrm{cu}}=R_{\mathrm{cu}}\left(I-I_{\mathrm{s}}\right)^{2} \\
& W_{\mathrm{s}}=v I_{\mathrm{s}}=R_{\mathrm{cu}}\left(I-I_{\mathrm{s}}\right) I_{\mathrm{s}} \\
& W_{\mathrm{T}}=W_{\mathrm{cu}}+W_{\mathrm{s}}=v I=R_{\mathrm{cu}}\left(I-I_{\mathrm{s}}\right) I .
\end{aligned}
$$

Nous traitons ici le cas particulier où le courant $I$ est égal à $I_{0}$. Il est intéressant d'introduire la température moyenne $\mathfrak{G}_{\mathrm{m}} \mathrm{du}$ supraconducteur. Elle s'exprime à partir des expressions [13], [14] mais encore directement en fonction du courant total $I_{\mathrm{s}}$ par

$$
I_{\mathrm{s}}=I_{0}\left(1-\frac{\mathfrak{C}_{\mathrm{m}}}{\mathfrak{C}_{\mathrm{c}}}\right) \text {. }
$$

La puissance totale à évacuer du composite pour le courant $I_{0}$ est alors tout simplement :

$$
W_{\mathrm{T}}=R_{\mathrm{cu}} I_{0}^{2} \frac{\mathfrak{G}_{\mathrm{m}}}{\mathfrak{G}_{\mathrm{c}}}
$$

En se souvenant que pour $I=I_{0}$,

$$
v=R_{\mathrm{cu}} I_{0} \frac{\mathfrak{C}_{\mathrm{m}}}{\mathfrak{G}_{\mathrm{c}}}
$$

en posant

$$
\beta_{\mathrm{d}}=\frac{R_{\mathrm{cu}} I_{0}^{2}}{8 \pi K_{\mathrm{s}} N \mathscr{G}_{\mathrm{c}}}
$$

on exprime la température $\mathscr{C}_{1}$ au bord du supraconducteur, ou du cuivre, c'est-à-dire celle intervenant dans le transfert, en fonction de la température moyenne $\mathscr{C}_{\mathrm{m}} \mathrm{du}$ supraconducteur

$$
\frac{\mathfrak{C}_{1}}{\mathfrak{C}_{\mathrm{c}}}=\frac{\mathfrak{G}_{\mathrm{m}}}{\mathfrak{C}_{\mathrm{c}}}-\left(1-\frac{\mathfrak{C}_{\mathrm{m}}}{\mathfrak{C}_{\mathrm{c}}}\right) /\left(1+\frac{1}{\beta_{\mathrm{d}}\left(\mathfrak{C}_{\mathrm{m}} / \mathscr{C}_{\mathrm{c}}\right)}\right) \text {. }
$$

5.3 Rôle DU FACTEUR DE STABILITÉ $\beta_{\mathrm{d}}$. - Le coefficient $\beta_{\mathrm{d}}$ joue un rôle particulier. Pour que la stabilité soit assurée, il faut que $\beta_{\mathrm{d}}$ reste inférieur à 1 . En effet, si l'on représente l'écart de température $\mathfrak{C}_{\mathrm{m}}$ entre supraconducteur et hélium en fonction de l'écart $\mathcal{C}_{1}$ entre cuivre et hélium, tel qu'il s'exprime par la relation (24) (Fig. 7), on s'aperçoit d'une part, que pour $\beta_{\mathrm{d}}$ nul, la température du supraconducteur est bien celle du cuivre $\left(\mathfrak{C}_{\mathrm{m}}=\mathfrak{C}_{1}\right)$. C'est le cas où la

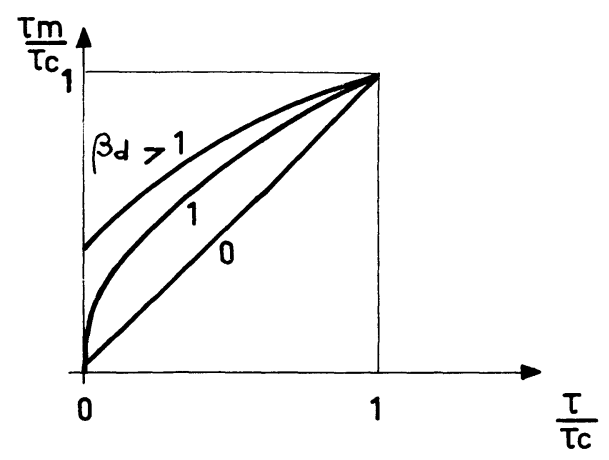

Fig. 7. - Température moyenne dans le supraconducteur.

conductibilité $K_{\mathrm{s}}$ serait infinie ou le diamètre des brins supraconducteurs nul. D'autre part pour toute autre valeur de $\beta_{\mathrm{d}}$, la température de supraconducteur est supérieure à celle du cuivre. En particulier pour $\beta_{\mathrm{d}}$ supérieur à 1 , il y a discontinuité et instabilité. La moindre augmentation de température $d \mathscr{C}_{1}$ du cuivre, entraîne l'apparition d'un régime instable où la puissance générée dans le supra

$$
\mathrm{d} W=R_{\mathrm{cu}} I_{0}^{2} \frac{\mathrm{d} \mathscr{C}_{\mathrm{m}}}{\mathfrak{C}_{\mathrm{c}}}\left(1-2 \frac{\mathscr{C}_{\mathrm{m}}}{\mathfrak{C}_{\mathrm{c}}}\right)
$$

ne peut être évacuée à travers le supra en raison des dimensions et de la conductibilité thermique insuffisante. Pour une telle puissance, le gradient thermique moyen ne peut être que $\mathrm{d} W / 8 \pi K_{\mathrm{s}} N$. Si $K_{\mathrm{s}}$ n'est pas assez grand, $\beta_{\mathrm{d}}=R_{\mathrm{cu}} I_{0}^{2} / 8 \pi K_{\mathrm{s}} N \mathcal{G}_{\mathrm{c}}$ est supérieur à 1 et la température croît pour atteindre un régime d'équilibre où la puissance évacuable équilibre la puissance à évacuer.

On peut remarquer que ce critère peut être trouvé sous une forme appelée alors "stabilité dynamique ", en résolvant l'équation complète de la chaleur, incluant la chaleur spécifique et le temps. La condition de stabilité imposant que la température dans le brin décroisse en fonction du temps, se ramène à la même expression de $\beta_{\mathrm{d}}$.

Ainsi, même dans le cas d'un transfert parfait entre cuivre et hélium, il subsiste une condition de stabilité liée à l'épaisseur des brins supraconducteurs

$$
\beta_{\mathrm{d}}<1 \text {. }
$$

Ce coefficient $\beta_{\mathrm{d}}$ s'écrit :

$$
\beta_{\mathrm{d}}=\frac{R_{\mathrm{cu}} I_{0}^{2}}{2 \pi K_{\mathrm{s}} N \mathscr{G}_{\mathrm{c}}} \quad \text { ou encore } \beta_{\mathrm{d}}=\frac{p_{\mathrm{cu}}}{8} \frac{J_{0}^{2} r_{\mathrm{s}}^{2}}{K_{\mathrm{s}} \mathscr{G}_{\mathrm{c}} X}
$$


$r_{\mathrm{s}}$ est le rayon d'un brin supraconducteur, et $K_{\mathrm{s}}$ la conductibilité thermique du supraconducteur.

Application numérique. - En prenant pour $K_{\mathrm{s}}$ la valeur de $1,2 \times 10^{-3} \mathrm{~W} / \mathrm{cm} \mathrm{K}$ [4] pour le niobiumtitane, on peut évaluer les valeurs du coefficient de stabilité dimensionnel $\beta_{\mathrm{d}}$ reportées tableau IV.

\section{TABLEAU IV}

Le critère est visiblement satisfait très facilement en diminuant le diamètre du brin supraconducteur

\begin{tabular}{|c|c|c|c|c|}
\hline Conducteur & BIM & B 11 & $61-33$ & $1000-4$ \\
\hline $1 \mathrm{~T}$ & 3,7 & 0,45 & $\overline{0,09}$ & 0,02 \\
\hline$\left.\beta_{\mathrm{d}}\right\} 6 \mathrm{~T}$ & 0,65 & 0,13 & 0,05 & 0,01 \\
\hline
\end{tabular}

\subsection{RECHERCHE D'UN DIAMÈTRE MAXIMUM « STABLE »} POUR LES BRINS SUPRACONDUCTEURS. - La relation de stabilité peut être écrite en faisant apparaître le diamètre du brin supraconducteur :

$$
\varphi_{\mathrm{s}}<\frac{1}{J_{0}} \sqrt{\frac{32 K_{\mathrm{s}} \mathscr{G}_{\mathrm{c}} X}{\rho_{\mathrm{cu}}}}
$$

de même qu'au chapitre 3.2 .3 , on peut déterminer en fonction du champ magnétique et du rapport cuivre/supra le diamètre maximum des brins supraconducteurs assurant la stabilité. Cette courbe est tracée figure 8 pour un rapport $X=A_{\text {cu }} / A_{\mathrm{s}}$ de 1 . Pour toute autre valeur du rapport cuivre/supra, il suffit de multiplier $\varphi_{\mathrm{s}_{1}}$ par $\sqrt{X}$.

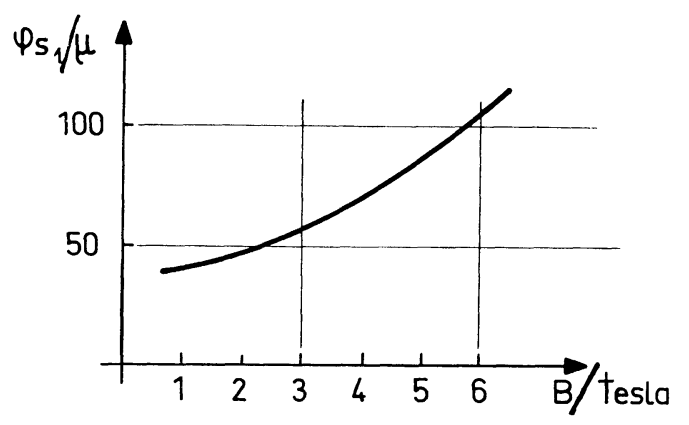

FIG. 8. - Diamètre " stable » du supraconducteur pour un rapport $X$ différent de 1 , il faut multiplier $\varphi_{\mathrm{s}_{1}}$ par $\sqrt{X}$.

Pour les champs faibles $(1 \mathrm{~T})$, on trouve $\varphi_{\mathrm{s}_{1}}=45 \sqrt{X}$ exprimé en microns.

5.5 CONDITIONS DE STABILITÉ POUR UN COMPOSITE EN FONCTION DE L'ÉPAISSEUR DES BRINS SUPRACONDUCTEURS ET DU TRANSFERT CUIVRE/HÉLIUM. - Il nous faut maintenant tenir compte du transfert de chaleur du cuivre vers l'hélium sur le diagramme puissance par unité de surface mouillée-écart de température. Il faut remplacer la droite de charge

$$
W=R_{\mathrm{cu}} I_{0}^{2} \frac{\mathfrak{C}}{\mathfrak{C}_{\mathrm{c}}}
$$

du brin fin, par une courbe

$$
W=f\left(\mathcal{G}_{1}\right) .
$$

Cette courbe est déduite de la relation :

$$
W=R_{\mathrm{cu}} I_{0}^{2} \frac{\mathfrak{G}_{\mathrm{m}}}{\mathfrak{G}_{\mathrm{c}}}
$$

et de l'expression (24) reliant $\mathfrak{C}_{1}$ à $\mathfrak{C}_{\mathrm{m}}$.

Elle est traçée sur la figure 9.

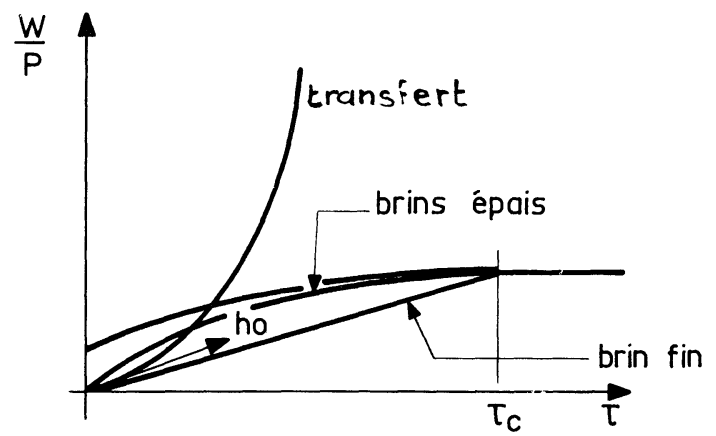

Fig. 9. - Détermination du point de fonctionnement.

Le point d'intersection avec la courbe de transfert donne le point de fonctionnement, et la pente à l'origine la condition de stabilité résumée à :

$$
\frac{\mathrm{d} W}{\mathrm{~d} \mathscr{C}_{1}} \cdot \frac{1}{h_{0} P}<1
$$

exprimant l'équilibre entre les puissances générée et évacuée.

On voit bien sur la figure 9 que pour les cas où $\beta_{\mathrm{d}}$ est supérieur à 1 , il apparaît une instabilité initiale même si le transfert est très bon.

Le développement de la condition (27) nous amène à

$$
\frac{\rho_{\mathrm{cu}} J_{0}^{2} \pi r_{\mathrm{s}}^{2}}{X \mathcal{G}_{\mathrm{c}}}\left[\frac{1}{8 \pi K_{\mathrm{s}}}+\frac{N}{h_{0} P}\right]<1
$$

qui peut s'écrire encore synthétiquement

$$
\beta_{\mathrm{t}}+\beta_{\mathrm{d}}<1
$$

les quantités $\beta_{\mathrm{t}}$ et $\beta_{\mathrm{d}}$ étant celles définies plus haut.

C'est-à-dire que non seulement les deux critères doivent être satisfaits indépendamment, mais encore la somme de deux. Il va sans dire que dans l'un ou l'autre cas tel ou tel facteur est prépondérant devant l'autre.

Ainsi pour les cas que nous avons déjà vus, rappelons à $6 \mathrm{~T}$ les résultats dans le tableau $\mathrm{V}$.

\section{TABLEAU V}

$\begin{array}{ccccc}\text { Conducteur } & \text { BIM } & \text { B 11 } & 61-33 & 1000-44 \\ \overline{\beta_{\mathrm{t}}} & \overline{0,6} & \overline{3,3} & \overline{2,2} & \overline{5} \\ \beta_{\mathrm{d}} & 0,6 & 0,13 & 0,05 & 0,01 \\ \text { Total } & 1,2 & 3,43 & 2,25 & 5,01\end{array}$


On voit que seul le conducteur « BIM » très stabilisé assure pratiquement la stabilité, quant aux autres, le critère de dimension du brin est très largement satisfait.

6. Rôle joué par une épaisseur de cuivre importante. - Il n'est pas superflu d'estimer le gradient thermique provenant d'une épaisseur importante de cuivre. On sent bien qu'à rapport cuivre sur supraconducteur donné, il n'est pas indifférent d'avoir un conducteur de petit ou de grand diamètre. Nous supposons d'abord tout le supraconducteur localisé au centre avec un rayon $R_{\mathrm{s}}=r_{\mathrm{s}} \sqrt{N}$ (Fig. 10 ; soit $W_{\mathrm{s}}$ la puissance dans le supraconducteur à une température $\mathfrak{C}$ (isotherme) et $W_{\text {cu }}$ la puissance dans le cuivre.

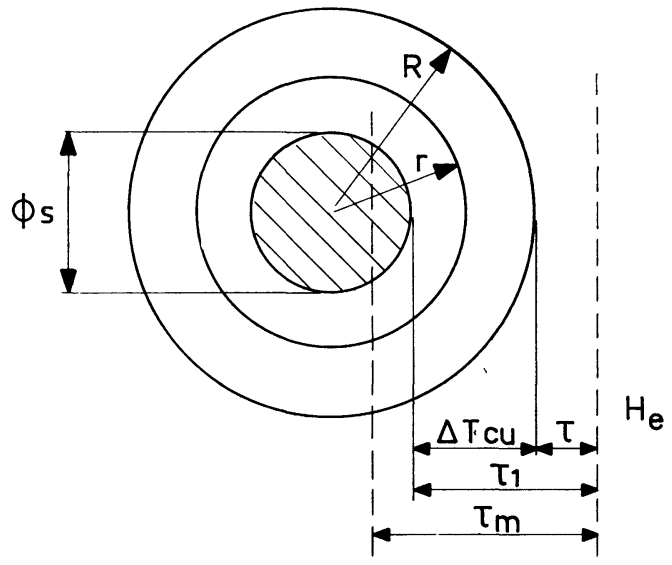

FIG. 10. - Influence de l'épaisseur de cuivre

L'équation de la chaleur permet d'écrire, à la distance $r$ du centre

$-2 \pi r K_{\mathrm{cu}} \frac{\partial T}{\partial r}=W_{\mathrm{s}}+\pi\left(r^{2}-R_{\mathrm{s}}^{2}\right) \frac{W_{\mathrm{cu}}}{\pi\left(R^{2}-r_{\mathrm{s}}^{2}\right)}$.

Equation qui conduit à :

$K_{\mathrm{cu}} \Delta T_{\mathrm{cu}}=\frac{1}{2 \pi} \log \frac{R}{R_{\mathrm{s}}}\left\{W_{\mathrm{s}}-W_{\mathrm{cu}} \frac{R_{\mathrm{s}}^{2}}{R^{2}-R_{\mathrm{s}}^{2}}\right\}+\frac{W_{\mathrm{cu}}}{4 \pi}$ ou encore si l'on fait apparaître le rapport $X$ :

$$
\begin{aligned}
& K_{\mathrm{cu}} \Delta T_{\mathrm{cu}}= \\
= & \frac{W_{\mathrm{s}}}{4 \pi} \log (1+X)+\frac{W_{\mathrm{cu}}}{4 \pi}\left\{1-\frac{\log (1+X)}{X}\right\} .
\end{aligned}
$$

6.1 Il est intéressant de comparer le gradient thermique dans le cuivre au gradient thermique dans un brin supraconducteur en raison des conductibilités thermiques très différentes. Lors du partage de courant pour une élévation de température $\mathcal{C}$ faible, la puissance dans le cuivre reste faible par rapport à la puissance dans le supraconducteur qui représente pratiquement la puissance totale

$$
W_{\mathrm{T}}=R_{\mathrm{cu}} I_{0}^{2} \frac{\mathscr{C}_{\mathrm{m}}}{\mathscr{C}_{\mathrm{c}}}
$$

$$
\begin{aligned}
& W_{\mathrm{s}}=R_{\mathrm{cu}} I_{0}^{2} \frac{\mathfrak{C}_{\mathrm{m}}}{\mathfrak{G}_{\mathrm{c}}}\left(1-\frac{\mathfrak{C}_{\mathrm{m}}}{\mathfrak{C}_{\mathrm{c}}}\right) \\
& W_{\mathrm{cu}}=R_{\mathrm{cu}} I_{0}^{2}\left(\frac{\mathscr{C}_{\mathrm{m}}}{\mathfrak{C}_{\mathrm{c}}}\right)^{2}
\end{aligned}
$$

donc

$$
K_{\mathrm{cu}} \Delta T_{\mathrm{cu}} \simeq \frac{W_{\mathrm{s}}}{4 \pi} \log (1+X)
$$

tandis que, en première approximation, on peut écrire dans un brin supraconducteur :

$$
K_{\mathrm{s}} \Delta T_{\mathrm{s}} \simeq \frac{W_{\mathrm{s}}}{4 \pi N}
$$

Soit

$$
\frac{\Delta T_{\mathrm{cu}}}{\Delta T_{\mathrm{s}}} \simeq \log (1+X) \frac{K_{\mathrm{s}}}{K_{\mathrm{cu}}} N
$$

Ainsi, pour un petit nombre de brins (inférieur à $1000)$ le gradient thermique sur le cuivre est négligeable, par contre pour $N$ supérieur à 1000 , il ne peut être négligé (pour le $1000-44$ on trouve ainsi $\left.\Delta T_{\mathrm{cu}} / \Delta T_{\mathrm{s}} \sim 0,3\right)$.

6.2 INFLUENCE COMBINÉE DE L'ÉPAISSEUR DU CUIVRE, DU TRANSFERT CUIVRE/HÉLIUM ET DE L'ÉPAISSEUR DES BRINS SUPRACONDUCTEURS (Fig. 10). - C'est le cas le plus général du composite nu, baignant dans l'hélium liquide.

Reprenons l'expression de la puissance totale dans le composite

$$
W_{\mathrm{B}}^{\prime}=R_{\mathrm{cu}} I_{0}^{2} \frac{\mathfrak{C}_{\mathrm{m}}}{\mathfrak{C}_{\mathrm{c}}}
$$

Le gradient thermique dans le cuivre s'écrit :

$$
K_{\mathrm{cu}} \Delta T_{\mathrm{cu}}=\frac{R_{\mathrm{cu}} I_{0}^{2}}{4 \pi} \frac{\mathfrak{G}_{\mathrm{m}}}{\mathfrak{G}_{\mathrm{c}}} \log (1+X) .
$$

On peut écrire $\mathcal{C}$ lié à la température au bord du cuivre en fonction de $\mathfrak{C}_{1}$ au bord du supraconducteur

$$
\mathfrak{C}=\mathfrak{C}_{1}-\Delta T_{\text {cu }} \text {. }
$$

En accord avec les relations (13) et (25) $\mathcal{G}$ s'écrit :

$$
\mathfrak{C}=\mathscr{C}_{\mathrm{m}}\left[1+\frac{\beta_{\mathrm{d}}\left(\frac{\mathscr{C}_{\mathrm{m}}}{\mathfrak{C}_{\mathrm{c}}}-1\right)}{\beta_{\mathrm{d}}\left(\frac{\mathscr{C}_{\mathrm{m}}}{\mathfrak{C}_{\mathrm{c}}}+1\right)}-\beta_{\mathrm{cu}}\right]
$$

si l'on appelle $\beta_{\text {cu }}$ l'expression

$$
\frac{\rho_{\mathrm{cu}} J_{0}^{2} R^{2} \log \left(1^{\top}+X\right)_{! !}}{4 K_{\mathrm{cu}} \mathcal{G}_{\mathrm{c}} X(1+X)}
$$

Le fait que $\mathcal{C}$ ne peut être négatif exige que le coefficient $\beta_{\mathrm{cu}}$ soit au moins inférieur à 1 . Un coefficient de stabilité $\beta_{\text {cu }}$ propre à l'épaisseur de cuivre est ici mis en évidence. La conductibilité du cuivre et le diamètre 
extérieur doivent être tels que la chaleur puisse être encore évacuée vers le bain.

Une application numérique conduit au rapport cuivre/supra donné à un diamètre maximum de l'ordre de $2,5 \mathrm{~mm}$ à multiplier par l'expression

$$
\sqrt{\frac{X(1+X)}{\log (1+X)}},
$$

c'est-à-dire par 1,7 pour $X=1$ et par 2,5 pour $X=2$. Le critère est donc très facilement satisfait, mais il y a risque pour les conducteurs massifs trop gros. La condition générale de stabilité assurée par l'inégalité de base $\mathrm{d} W / \mathrm{d} C<h_{0} P$ conduit à :

$$
\frac{R_{\mathrm{cu}} I_{0}^{2}}{h_{0} P \cdot \mathscr{G}_{\mathrm{c}}} \frac{1}{1-\beta_{\mathrm{cu}}-\beta_{\mathrm{d}}}<1 \text {. }
$$

Soit encore :

$$
\frac{\beta_{\mathrm{t}}}{1-\beta_{\mathrm{cu}}-\beta_{\mathrm{d}}}<1 \text {. }
$$

Si le transfert est très bon, la condition se ramène à $\beta_{\mathrm{d}}+\beta_{\mathrm{cu}}<1$ elle-même pratiquement limitée à la condition liée à l'épaisseur des brins supraconducteurs. Ainsi l'épaisseur de cuivre joue par l'intermédiaire du gradient thermique un rôle généralement négligeable, sauf pour les composites à très grand nombre de filaments.

Nota. - Le coefficient lié au cuivre s'écrit encore :

$$
\beta_{\mathrm{cu}}=\frac{\rho_{\mathrm{cu}} J_{0}^{2} R_{\mathrm{s}}^{2} \log (1+X)}{4 K_{\mathrm{cu}} X \mathcal{G}_{\mathrm{c}}} .
$$

7. Influence d'une couche isolante. - Généralement, les conducteurs sont recouverts d'une mince couche isolante (formvar, résine, vernis, mylar, etc...). Si les conditions de transfert à l'hélium sont difficiles à connaître, on peut cependant s'apercevoir que la mauvaise conductibilité thermique de cet isolant peut être source d'instabilités supplémentaires.

Si $W$ est la puissance émise dans le composite (Fig. 11), la différence de température sur l'épaisseur d'isolant $\Delta T_{\mathrm{i}}$ peut s'écrire :

$$
\Delta T_{\mathrm{i}}=\frac{W}{2 \pi K_{\mathrm{i}}} \log \frac{R_{\mathrm{i}}}{R}
$$

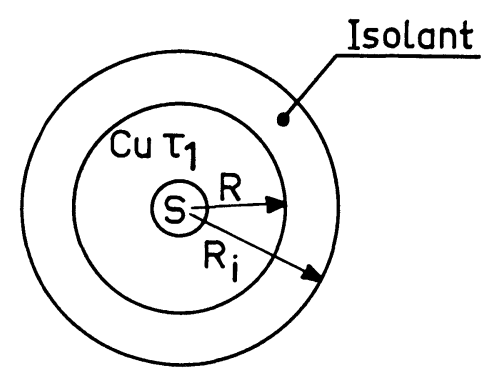

Fig. 11. - Influence de l'épaisseur d'isolant. si $\varepsilon$ est l'épaisseur d'isolant et $P$ le périmètre mouillé, il vient :

$$
\Delta T_{\mathrm{i}}=\frac{W}{P} \cdot \frac{\varepsilon}{K_{\mathrm{i}}}
$$

analogue à un transfert.

Soit $T$ la température extérieure de l'isolant

$$
\left(T=T_{0}+\mathcal{G}\right),
$$

$T_{1}$ la température du composite supposé isotherme $\left(T_{1}=T_{0}+\mathcal{C}_{1}\right)$, on peut écrire :

$$
\mathfrak{C}_{1}=\mathscr{C}+\frac{W}{P} \frac{\varepsilon}{K_{\mathrm{i}}}
$$

avec

$$
W=R_{\mathrm{cu}} I_{0}{ }^{2} \frac{\mathfrak{G}_{1}}{\mathfrak{G}_{\mathrm{c}}}
$$

soit

$$
\mathscr{C}=\mathscr{C}_{1}\left[1-\frac{R_{\mathrm{cu}} I_{0}^{2}}{\mathscr{C}_{\mathrm{c}}} \frac{\varepsilon}{P K_{\mathrm{i}}}\right]
$$

Cette expression montre clairement :

1) que la température $T_{1}$ du composite Test nettement augmentée par rapport à la température】 $T$ extérieure de l'isolant,

2) que la courbe de transfert en présence d'isolant est décalée vers les températures plus élevées (sans pour autant pouvoir la déduire car les conditions d'échange sont différentes) et de ce fait, un système stable à cuivre nu peut devenir instable avec isolant (Fig. 12),

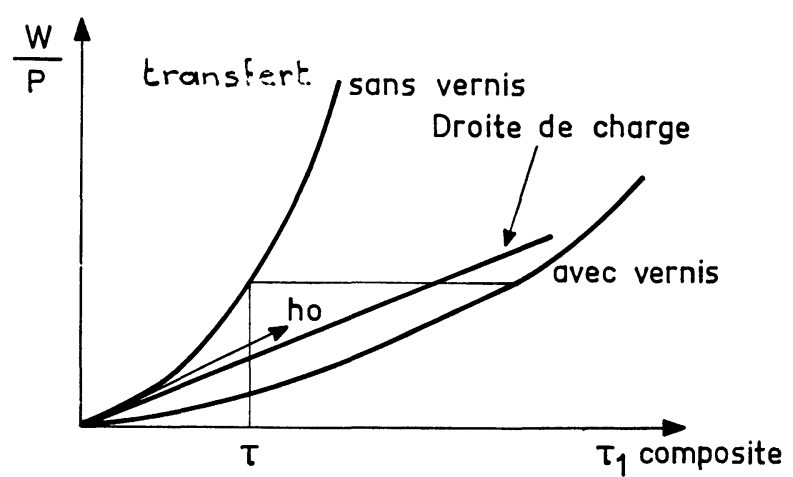

Fig. 12. - Influence de l'isolant sur la courbe de transfert.

3) qu'une instabilité fondamentale propre à l'isolant peut de toute façon apparaître si l'expression en facteur devant $\mathfrak{C}_{1}$ est nulle ou négative.

Ainsi, on peut définir un critère de stabilité lié à l'isolant

$$
\underline{\beta_{\mathrm{i}}<1}
$$

avec

$$
\beta_{\mathrm{i}}=\frac{R_{\mathrm{cu}} I_{0}^{2} \varepsilon}{\mathcal{G}_{\mathrm{c}} P K_{\mathrm{i}}}
$$


ou encore

$$
\beta_{\mathrm{i}}=\frac{\rho_{\mathrm{cu}} J_{0}^{2} \varepsilon R}{2 X(1+X) K_{\mathrm{i}} \mathcal{\zeta}_{\mathrm{c}}} .
$$

Si l'on connaît le coefficient de transfert isolanthélium $h_{0 \mathrm{i}}$, on peut considérer que l'isolant intervient par l'intermédiaire d'un coefficient de transfert $h_{\mathrm{i}}=K_{\mathrm{i}} / \varepsilon$. Le transfert ramené au composite s'écrit :

$$
\mathcal{G}_{1}=\frac{W}{P}\left[\frac{1}{h_{0 \mathrm{i}}(\mathfrak{G})}+\frac{\varepsilon}{K_{\mathrm{i}}}\right] \text {. }
$$

Application numérique. - Pour $I=I_{0}$, et pour un écart de température $\mathcal{C}$ très petit, il est intéressant de voir qu'une couche d'isolant de $25 \mu$ d'épaisseur affectée d'une conductibilité thermique de $10^{-3} \mathrm{~W} / \mathrm{cm} \mathrm{K}$ est équivalente à un transfert thermique supplémentaire de $0,4 \mathrm{~W} / \mathrm{cm}^{2} \mathrm{~K}$, c'est-à-dire le cas pris au paragraphe 3.2.2 et que dans ces conditions particulières, on trouve pour $\beta_{\mathrm{i}}$ les mêmes valeurs que $\beta_{\mathrm{t}}$.

8. Remarque sur la réponse d'un conducteur composite à une perturbation. - -8.1 TRANSFERT EN IMPULSION. - L'analyse cryostatique suppose la perturbation initiale terminée. Un régime permanent est établi pour lequel on peut parler de transfert. Or, comme nous le verrons plus loin, la nature, l'importance et la durée de l'impulsion perturbatrice sont fondamentales. Des études ont été menées en ce sens pour étudier la réponse du système [16] à une perturbation. Or, on sait déjà [17] que le coefficient d'échange thermique $h_{0}$ est bien amélioré pendant les premières millisecondes de la perturbation (par exemple, on peut dissiper $1,2 \mathrm{~W} / \mathrm{cm}^{2}$ pendant $0,5 \mathrm{~ms}$ ). Ce phénomène va ainsi dans le sens d'une amélioration de la stabilité du système.

8.2 Rôle ENTHALPIQUE DE L'HÉLIUM. - En raison de la très grande chaleur latente de vaporisation de l'hélium $(24,5 \mathrm{~J} / \mathrm{g})$, une perturbation de courte durée peut être « encaissée » par l'hélium; la limite étant l'énergie totale de la perturbation, la montée en puissance du composite et la durée eu égard au volume de liquide à vaporiser, celui-ci étant rapidement limité en raison de la conductibilité thermique relativement faible du gaz formé. Cependant, ce système de stabilisation peut raisonnablement être envisagé. Un calcul très approché indique qu'une couche de $100 \mu$ d'hélium liquide autour d'un câble de $2 \mathrm{~mm}$ peut permettre de limiter la montée propre en température pendant $1 / 100$ de seconde.

9. Application au cas réel d'un composite où la tension apparaît de Imanière progressive. - Ainsi que nous l'avons rappelé au paragraphe 2.5 , il existe une zone où la tension croît très lentement avec le courant. On ne peut donc, en pratique, définir de courant $I_{0}$. Ainsi pour le conducteur $1000-44$ pour- tant très instable cryostatiquement, il est possible et facile d'observer l'apparition de la tension au-dessus du courant " critique ". Il existe en fait une zone de stabilité « conditionnelle » comme nous allons le voir.

9.1 RôLE DE LA RÉSISTANCE DIFFÉRENTIELLE POUR LES FAIBLES PERTURBATIONS EN TEMPÉRATURES. - Supposons, représentées figure 13 , les caractéristiques $v(I)$ réelles pour des isothermes $0, \mathfrak{C}_{1}, \mathfrak{C}_{2}$. Le long d'une courbe $v(I)$, si $I$ est suffisamment supérieur à $I_{0}$, il y a régime de partage de courant et la pente $\mathrm{d} v / \mathrm{d} I$ devient pratiquement égale à $R_{\mathrm{cu}}$.

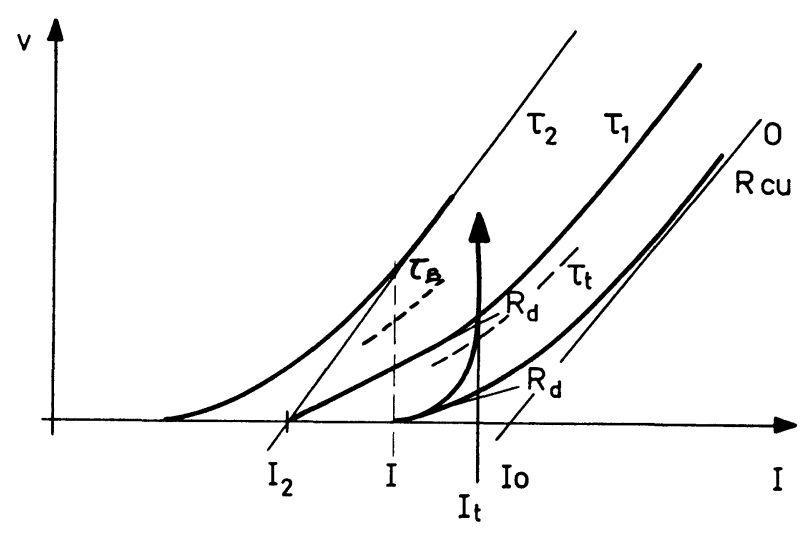

Fig. 13. - Influence de la température sur la courbe $v(I)$.

Plaçons-nous à la température du bain $T_{0}$ à un courant $I$ inférieur à $I_{0}$, tel que la tension reste très faible et la puissance insuffisante pour chauffer notablement. Chauffons artificiellement ; lorsque $\mathscr{C}$ croît, $I$ restant constant, le point de fonctionnement se déplace verticalement sur le réseau isotherme. En tout point, on a :

$$
W=v I
$$

avec

$$
v=R_{\mathrm{d}}[\Delta I]_{0}^{\mathscr{C}} \simeq R_{\mathrm{d}} I \frac{\mathscr{C}}{\mathfrak{G}_{\mathrm{c}}}
$$

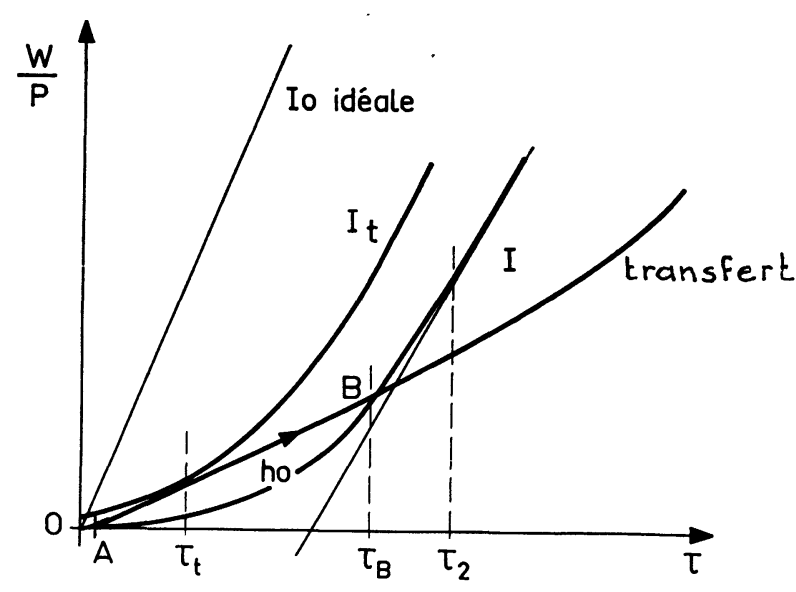

Fig. 14. - Courbe de charge réelle $W(\mathfrak{C})$ et transfert à l'hélium. 
Donc :

$$
W=R_{\mathrm{d}} I^{2} \frac{\mathscr{G}}{\mathfrak{G}_{\mathrm{c}}},
$$

c'est-à-dire que la résistance différentielle $R_{\mathrm{d}}$ joue le même rôle pour les petites perturbations $\mathcal{C}$ que la résistance du cuivre pour les grandes. Il est donc intéressant de suivre continûment ce qui se passe lorsque $\mathscr{C}$ croissant, $R_{\mathrm{d}}$ croît jusqu'à atteindre $R_{\mathrm{cu}}$. La puissance émise croît comme le produit $R_{\mathrm{d}}(v, \mathcal{C}) . \mathcal{C}$ jusqu'à ce que soit atteint le régime de partage à la température $\mathfrak{C}_{2}$ où $R_{\mathrm{d}}=R_{\mathrm{cu}}$. A la droite de charge $R_{\mathrm{cu}} I_{0}^{2} \mathfrak{C} / \mathcal{G}_{\mathrm{c}}$ s'est donc substituée une courbe à pente très faible à l'origine qui se redresse progressivement pour venir prendre l'allure d'une droite (Fig. 14).

Lorsque pour obtenir la caractéristique $v(I)$ le courant est augmenté progressivement, la température d'équilibre reste, tout d'abord, voisine de celle du bain pendant que la courbe de charge se déplace vers le haut, ramenant progressivement le point $B$ instable vers le point de fonctionnement A stable et proche de l'origine. Lorsque les deux se confondent, le système transite pour un courant $I_{\mathrm{t}}$ et une température $\tau_{t}$ (Fig. 13, 14).

9.2 SÉCURITÉ EN TEMPÉRATURE. - Le rôle de ce point $B$ est très important. En effet, si l'on fonctionne à un courant $I$ où la tension commence à apparaître et bien que le composite ne soit pas stable (vis-à-vis de la résistance du cuivre), il existe, du fait de la courbure, une sécurité en température. Le composite peut encaisser des montées en température inférieures à $\mathcal{C}_{\text {B }}$ sans entraîner de blocage. La stabilité est condi- tionnée à l'amplitude de la perturbation en température.

L'ordre de grandeur de cette garde en température peut être estimé. Ainsi, pour le $1000-44$, on possède une marge d'une dizaine d'ampères avant blocage. Si l'on fixe le courant de fonctionnement $I$ à $100 \mathrm{~A}$, on peut encaisser une variation de $10 \%$, c'est-à-dire au minimum une garde de température de $0,1 \mathscr{C}_{c}$ soit $0,2 \mathrm{~K}$ à $6 \mathrm{~T}$.

10. En guise de conclusion sur la stabilisation cryostatique. - Nous noterons le rôle important joué par l'amplitude de la perturbation initiale en température.

a) Stabilité conditionnelle. $-\mathrm{Si} \mathscr{C}$ est petit $\left(\mathscr{C}<\mathscr{C}_{\mathbf{B}}\right)$, on applique les relations précédentes en remplaçant $R_{\text {cu }}$ par $R_{\mathrm{d}}$. Celle-ci étant en général au moins 100 fois plus faible que celle du cuivre, le composite est pratiquement stable.

D'où l'importance d'un conducteur adiabatiquement ou intrinsèquement stable et bien maintenu.

b) Stabilité fondamentale. - Si $\mathcal{C}$ est grand (mouvements importants par exemple), c'est-à-dire si $\mathscr{C}>\mathfrak{C}_{\mathbf{B}}$ on applique les relations avec la résistance du cuivre.

On voit, ainsi, clairement toute l'importance de limiter au maximum l'amplitude des perturbations thermiques en empêchant dès leur source toute instabilité d'origine magnétique ou mécanique. En ce qui concerne ces dernières, dans le cas de bobinages de grand volume à structure complexe, il est particulièrement difficile d'empêcher totalement l'apparition de perturbations importantes. Il n'y a, alors, d'autre ressource pour assurer la stabilité que d'augmenter la quantité de cuivre pour satisfaire les critères ci-dessus énoncés.

\section{Bibliographie}

[1] Wilson, M. N., Walters, C. E., Lewin, J. D., Smith, P. F., SPURWAY, A. H., Report RPP/A 73, Rutherford (1969).

[2] Chester, P. F., Rep. Prog. Phys. 30 (1967) 561.

[3] HLASNIK, I., Echanges thermiques dans l'hélium I, CEN Saclay (1970), rapport interne DSS/GATS 70-18.

[4] Février, A., Sagniez, A., Turck, B., Vesvre, E., CEN Saclay, rapport interne SEDAP/73-27 MES (1973).

[5] Efferson, K. R., J. Appl. Phys. 40 (1969) 1995.

[6] Gauster, W. F., J. Appl. Phys. 40 (1969) 2060.

[7] Stekly, Z. J. J., ZAR, J. L., IEEE Trans. on Nucl. Sc. (1965) NS 12, 367.

[8] Stekly, Z. J. J., Proceedings of the 1st Int. Symposium on Magnet Technology (Stanford) 1965 p. 550.

[9] Stekly, Z. J. J., Thomas, R., Strauss, B., Proceedings of the 1968 summer study on superconductor devices (Brookhaven) 1968 p. 748.

[10] MadDOCK, B. J. et al., Cryogenics 9 (1969) 261.

[11] Carter, C. N., Lewis, K. G., Maddock, B. J., Cryogenics 13 (1973) 337.

[12] Wilson, M. N., Proceedings of the 2nd Conference on Magnet Technology (Oxford) (1967) p. 482.

[13] Lubell, M. S., Kroeger, D. M., Advances in Cryog. Engineering 14123.

[14] SAgniez, A., Turck, B., Vesvre, E., CEN Saclay, rapport interne SEDAP/73-31 MES (1973).

[15] FÉVrier, A., TURCK, B., CEN Saclay, rapport interne SEDAP/72-41 MES (1972).

[16] Iwasa, Y. et al., J. Appl. Phys. 40 (1969) (2006).

[17] JACKson, J., Cryogenics 9 (1969) 103. 